\title{
Inclusión educativa de personas sordas señantes en nivel universitario en México
}

\author{
Ulises Delgado Sánchez, Fernanda Gabriela Martínez Flores \\ Centro de Investigación Transdisciplinar en Psicología, UAEM \\ Alma Janeth Moreno Aguirre, Leticia Stephany Hernández Bucio \\ Facultad de Comunicación Humana, UAEM
}

\section{Resumen:}

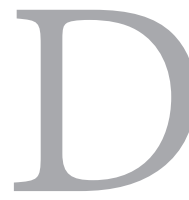

iversas universidades en México han comenzado a implementar la inclusión de personas con discapacidad, motriz y sensorial. Aún falta investigar más sobre las mejores prácticas de la inserción de los estudiantes sordos en universidad, desde la percepción de sus pares, hasta las practicas académicas y administrativas pertinentes, por ello, el presente capítulo tiene por objetivo explorar la percepción que tienen los estudiantes pares, profesores y personal administrativo de la Facultad de Comunicación Humana de la Universidad Autónoma del Estado de Morelos sobre la inclusión de estudiantes sordos en esa institución. Para ello se aplicó a 101 estudiantes y personal de la $\mathrm{FCH}$ cuestionarios tipo Likert, basados en el índice de inclusión de la UNESCO. Los resultados indican que todos los participantes están informados sobre las políticas inclusivas para estudiantes sordos, y la

\section{Summary:}

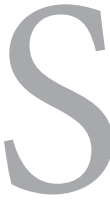

everal universities in Mexico have begun implementing the inclusion of people with motor and sensory disabilities. Still need more research on best practices for the inclusion of deaf students in college, from the perception of their peers, to the relevant academic and administrative practice, therefore, this chapter aims to explore the perception of peer students, faculty and staff of the School of Human Communication at the Autonomous University of the State of Morelos on the inclusion of deaf students at that institution. To this it was applied to 101 students and staff of the SHC Likert questionnaires based on the rate of inclusion of UNESCO. The results indicate that all participants are informed about inclusive policies for deaf students, and the importance of an inclusive culture, but also perceived barriers to learning and social 
importancia de una cultura inclusiva, pero también perciben barreras para el aprendizaje y barreras sociales, como es el paternalismo de los docentes y la falta de información a los estudiantes sordos. Finalmente, se esboza un plan de acción para abatir las barreras detectadas.

Palabras clave: inclusión, educación, sordos, universidad barriers, as is the paternalism of teachers and the lack of information to the deaf students. Finally, an action plan outlining the identified barriers to abate.

Keywords: inclusion, education, deaf, college

\section{Introducción}

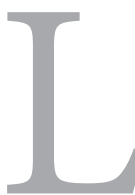

os cimientos del concepto de inclusión fueron establecidos por Boothy Ainscow (2000), definiéndolo como un conjunto de procesos orientados a eliminar o minimizar las barreras que limitan el aprendizaje y la participación de todos los estudiantes. Este término ha tenido impacto en los diferentes niveles y contextos educativos, vinculándose de manera sólida con las Necesidades Educativas Especiales (NEE) que hacen referencia a la discapacidad, la cual se define como la deficiencia física, mental, o sensorial ya sea de manera permanente o temporal, que limita la capacidad de ejercer una o más actividades esenciales de la vida diaria, que puede ser causada o agravada por el entorno económico y social (Andrade, Monsalve \& Aristizábal, 2015).
Referente a las personas con una deficiencia sensorial, específicamente la auditiva, se remontan sus antecedentes en la educación con Rudolf Agrícola (1443-1485) quien fue el primero en reportar el caso de una persona que de nacimiento era sorda y aprendió a leer y escribir (Díaz \& Rodríguez, 2009). Décadas posteriores Gerónimo Cardano (1501-1576) planteó las bases para la educación de las personas sordas bajo el principio de que "la vista sustituye al oído", enseñando el uso de signos manuales para que las personas sordas se comunicaran con los oyentes y podían adquirir la capacidad de expresarse de manera escrita y en algunos casos de manera oral (Jullian, 2001). Estos fueron los antecedentes de lo que actualmente se llama bilingüismo en los alumnos sordos, quienes desarrollan dos modalidades distintas de lenguas, la primera de ellas es la lengua de señas y la segunda es la lengua que la mayoría de la población 


\section{Artículos Empíricos}

usa, dependiendo de la región territorial que se encuentren (Lissi, Svartholm \& González, 2012). En el caso de México, el español, se adquiere en su modalidad escrita y en algunos casos oral.

En el Censo de Población y Vivienda 2010 se identificó que en ese año en México había $5,739,270$ personas con alguna limitación para realizar sus actividades cotidianas, presentando dificultades para escuchar un total de 694,464 mexicanos (Lara, 2013), y sordera, dos por cada cien habitantes (Cruz, 2013). En 2011 fue emitida la Ley General para la Inclusión de las Personas con Discapacidad, la cual establece la inclusión social, laboral y educativa (Lara, 2013). Acorde con la implementación de ésta normatividad la Secretaria de Educación Pública en México (2012) fundó algunos centros educativos en los que se empezó a implementar un modelo educativo que favorece la modalidad lingüística pertinente para comunicarse con los estudiantes sordos y cuentan con la participación de docentes y adultos sordos. Sin embargo, es imperante que las escuelas de educación básica ofrezcan espacios de inclusión, ambientes de aprendizaje y convivencia bilingüe y bicultural que permitan que los niños sordos crezcan y vivan comunicados. El avance en la educación básica ha sido muy lento. No basta con aprobar leyes si no construyen las condiciones culturales e institucionales, desde el estudiante, la familia, los docentes, directivos, administrativos (Delgado, Martinez, Flores \& Lechuga, 2016).
Es necesario superar algunas de las barreras del aprendizaje entre las que se encuentran: a) modelos a seguir, considerando aspectos curriculares de la organización o institución y una metodológica viable, b) falta de investigación en el tema que dé un sustento empírico para mejorar el proceso de inclusión, c) sensibilización de los profesionales educativos y trabajo colaborativo con un equipo multidisciplinario, d) estrategias de enseñanza y aprendizaje, e) descentralización de la educación (Céspedes, 2013; Gutiérrez, 2007; Soto, 2003; Valenzuela, Guillén \& Campa, 2014).

La inclusión de personas con NEE y en específico de personas con problemas auditivos no se ha universalizado en México, comienzan a surgir diversos intentos en las universidades para dar cobertura a esta población, entre las universidades que han implementadas prácticas diversas se encuentran:

- La Universidad Tecnológica de Tijuana; carrera de contaduría y administración e informática; las cuales elaboraron un material de apoyo didáctico, en la búsqueda de la inclusión e integración de la comunidad Sorda en el ámbito de la educación superior, por medio de un diccionario electrónico contable (Victoria, Arredondo \& Román, 2013).

- La Universidad Juárez del Estado de Durango (UJED) en la que han completados sus carrereas estudiantes sordos. El primero de ellos, en la Facultad de Psicología y Terapia de 
la Comunicación Humana, lo que permitió identificar algunos indicadores que se deben tener en cuenta para los programas educativos que buscan la inclusión de las personas sordas, se crearon cursos remedial para sordos, el intérprete y el asesor pedagógico; éste se creó con el objetivo de proveer el fortalecimiento y el desarrollo de las competencias en la escritura y lectura comprensivas del español, en la resolución de problemas matemáticos y en el uso tecnológico (TIC) con fines educativos y de investigación, posterior de tomar el curso los estudiantes pasan al curso propedéutico el cual al culminar les permitirá optar por una Licenciatura de su preferencia de acuerdo con sus capacidades y expectativas (Gómez, 2014). El segundo estudiante cursó la licenciatura en artes visuales, con un apoyo importante de la tutoría entre pares (el tutor es un estudiante oyente). Una información anecdótica derivada de este caso es lo que los docentes en un primer instante no sabían si debían dirigirse al intérprete o a los estudiantes, presentando incertidumbre, un ambiente tenso, intranquilo los primero días de clases. Esta investigación permitió definir un perfil de docente inclusivo (Ontiveros, 2015).

- La Universidad Tecnológica de Santa Catarina, Nuevo León (2013) en 2004 implemento un programa con el propósito de incluir en la matricula a personas con discapacidad, en la inclusión de personascon discapacidad auditiva ha implementado un modelo computacional con la finalidad de facilitar el proceso de selección de personal con esta característica, que permite la sustitución del intérprete de lengua de señas. La universidad cuenta con un grupo especializado de intérpretes dedicados a la impartición de la enseñanza de la lengua de señas mexicana a través de cursos, talleres y clases que tienen el propósito de difundir y promover el uso de la misma, el cual está dirigido a personal de la universidad y padres de familia oyentes y sordos.

- La Universidad de Guadalajara (2010) en su plan de desarrollo institucional 2014-2030 ha propuesto como objetivo ampliar la cobertura educativa a través de modalidades no convencionales pretendiendo diversificar los programas de educación continua mediante modalidades no convencionales, fortalecer el ingreso de estudiantes provenientes de grupos indígenas y desprotegidos mediante la incorporación de criterios equitativos de ingreso y permanencia de estudiantes.

- La Universidad Tecnológica del Valle del Mezquital del Estado de Hidalgo (2015) establece que toda persona con discapacidad que haya egresado del nivel medio superior puede ingresar a estudiar una carrera profesional, brindando un asesor académico par como apoyo, así como en el caso de los 


\section{Artículos Empíricos}

estudiantes sordos un intérprete de Lengua de Señas en sus clases y asesorías.

- La Universidad Autónoma del Estado de Morelos (UAEM) (2015) ha creado en el 2015 las Unidades de Servicio de Apoyo a la Inclusión Educativa (USAIE) para propiciar el desarrollo académico de los estudiantes con discapacidad, siendo sus principales funciones las de proporcionar tutorías y seguimiento académico de los alumnos, seguimiento y asesoría a unidades académicas, asesoría psicopedagógica a los docentes, estipulando que en los casos de sordera es necesario considerar la Lengua de Señas Mexicana.

En el caso que nos atañe que es la inclusión en la UAEM, aun y cuando se han propuesto las USAIE se presenta un reto en realizar un estudio diagnóstico de las necesidades académicas e institucionales que son necesarias para la inclusión de estudiantes sordos señantes. Debido a la movilidad de una estudiante sorda señante de la UJED que acudió con su intérprete a la Facultad de Comunicación Humana $(\mathrm{FCH})$ de la UAEM ubicada en la Ciudad de Cuernavaca, Morelos, México, se realizó un estudio de carácter cualitativo, exploratorio descriptivo, transversal acerca del conocimiento de prácticas inclusivas en el personal administrativo, el personal docente y en la población estudiantil con relación a la inclusión educativa de estudiantes sordos señantes durante el semestre agosto-diciembre del 2015. El objetivo del trabajo fue identificar la percepción de estudiantes oyentes de la $\mathrm{FCH}$ respecto a las prácticas de inclusión de personas sordas en la facultad, así como identificar la percepción de los docentes y personal administrativo sobre la implementación de programas de inclusión de personas sordas y la situación actual en la que se encuentra su unidad académica referente a éstas prácticas.

\section{Método}

\section{Participantes}

La muestra seleccionada correspondió a estudiantes de licenciatura de la FCH de la UAEM pertenecientes a $4^{\circ}, 5^{\circ}$ y $7^{\circ}$ semestres (un total de 81 estudiantes), grupos donde la estudiante sorda señante tomó algunas asignaturas, así como también se integró una muestra de docentes que le impartieron clases a la alumna de movilidad y otros docentes que conformaron la planta docente de ese semestre y administrativos de diferentes áreas como son servicios escolares, personal de secretaría académica, del área de computo, de intendencia, etc. (un total de 20).

\section{Materiales}

Se aplicaron dos cuestionarios tipo Likert con cuatro opciones de respuesta, el primero de ellos se aplicó a los pares (estudiantes oyentes) (ver anexo 1) y el otro al personal docente y administrativo (ver anexo 2). Ambos se basaron 
en el Índice de inclusión (Booth \& Ainscow, 2000), tomando en cuenta las siguientes categorías para los estudiantes: políticas escolares, cultura escolar inclusiva, barreras para el aprendizaje, barreras sociales y participación, además de, principios de educación inclusiva para docentes y administrativos, como se describe a continuación:

a) Políticas escolares: analizan si se están implementando políticas escolares en la facultad para que se hagan adecuaciones en el programa educativo de la licenciatura en Comunicación Humana y se logre una igualdad de condiciones en el estudiante sordo señante con sus pares oyentes, está conformado por los reactivos 1,2,3,7,15

b) Cultura escolar inclusiva: identifica si en la facultad existe una comunidad escolar comprometida con la inclusión de los alumnos Sordos, donde cada alumno es valorado como fundamento primordial pese a la diversidad de características y necesidades que pudiesen presentar, lo cual favorece la convivencia armónica, respetuosa y tolerante para el derecho a la igualdad entre todos los agentes que convergen en la institución, está conformado por los reactivos 4,5,12,13

c) Barreras para el aprendizaje y la participación: corresponde a identificar cuáles son las principales barreras que los estudiantes oyentes consideran que se presentan como un obstáculo para el desarrollo óptimo del estudiante sordo, está conformado por los reactivos $6,8,10,14$

d) Barreras sociales: indaga sobre la aceptación social por parte de los pares de los estudiantes que presentan sordera dentro del ámbito educativo, está conformado por los reactivos 9 y 11

Se realizó el análisis estadístico para medir la validez y fiabilidad de los instrumentos aplicados con el programa IBM SPSS versión 21 para Windows. El cuestionario aplicado a los alumnos presento un Alfa de .67 y está conformado por 15 ítems; el cuestionario de docentes y personal administrativo presento un Alfa de .87 con 10 ítems

\section{Resultados}

Los cuestionarios se analizaron por grupo de participantes y por las categorías contempladas en el Índex. La población de estudiantes presentó un rango de edades entre los 19 a 33 años, con una mayor frecuencia en las edades de 20 a 21 años en un $58 \%$, así como predominó el sexo femenino en un $88.9 \%$ en relación al masculino en un $11.1 \%$. La población de docentes y administrativos que se integró a la muestra tuvo un rango de edad entre los 22 a los 55 años de edad, de igual manera predominó el sexo femenino en un $65 \%$ en relación al masculino con un 35\%. 


\section{Artículos Empíricos}

Los resultados globales por dimensión (Ver tabla1) reflejan que existe una percepción favorable de implementación de políticas escolares para la inclusión de estudiantes sordos, existiendo en la FCH una alta cultura escolar inclusiva, aun y cuando estas primeras dimensiones son favorables existe la percepción de una alta presencia de barreras para el aprendizaje y la participación que indica que aún faltan cosas por hacer para lograr que la FCH sea inclusiva en su totalidad; por lo que dentro de las barreras de aprendizaje que se hicieron evidentes, es que se tiende a caer en paternalismo con el estudiante sordo señante, sin valorar sus posibilidades y capacidades por lo que en ocasiones los docentes tienden a ser más flexibles a la hora de exigir la adquisición de competencias de las asignaturas; en las barreras sociales se consideró que la presencia del estudiante sordo señante en la facultad, no genera ningún rechazo por tener una condición diferente y que existe una alta aceptación hacia la Sordera, por lo que muestra que se tiene claro que la diversidad lejos de ser un problema es una oportunidad de aprendizaje para todos.

Unavezrevisadoslos resultados de las diferentes dimensiones de las encuestas se hace evidente la necesidad de trabajar para disminuir las barreras de aprendizaje y participación, ya que a manera general todos los encuestados evidenciaron que la FCH si es inclusiva en el sistema educativo sin embargo hay temas que no quedan muy claros y son ambiguos al no saber cómo organizar las actividades y la forma de incluir a la población sorda en la dinámica grupal.

Tabla 1

Puntajes globales por dimensión en la encueta a pares

\begin{tabular}{l|c|c|c|c}
\multicolumn{1}{c|}{ Dimensión } & $\begin{array}{c}\text { Totalmente de } \\
\text { Acuerdo }\end{array}$ & Acuerdo & Desacuerdo & $\begin{array}{c}\text { Totalmente en } \\
\text { Desacuerdo }\end{array}$ \\
\hline $\begin{array}{l}\text { Políticas escolares } \\
\text { Cultura escolar inclusiva }\end{array}$ & $29.58 \%$ & $25.67 \%$ & $28.36 \%$ & $16.38 \%$ \\
$\begin{array}{l}\text { Barreras para el aprendizaje } \\
\text { y la participación }\end{array}$ & $29.77 \%$ & $11.80 \%$ & $11.80 \%$ & $9.62 \%$ \\
Barreras sociales & $40.12 \%$ & $35.80 \%$ & $14.50 \%$ & $19.75 \%$ \\
\hline
\end{tabular}


La encuesta al personal docente $y$ administrativo evaluó la categoría de principios de educación inclusiva, donde se midió el potencial y características de la escuela en la que laboran para determinar si cuenta con el perfil de ser incluyente.

De los diez aspectos evaluados (ver Tabla 2) de forma general sólo en cuatro de ellos la tendencia fue hacia la postura totalmente de acuerdo y de acuerdo, esto indica que los docentes y personal administrativo tienen la percepción que la Universidad Autónoma del Estado de Morelos da apertura a programas de formación de docentes especializados en educación bilingüe para Sordos, que es necesario hacer adaptaciones curriculares a los programas, así como la $\mathrm{FCH}$ tiene una actitud inclusiva y previene la discriminación a estudiantes sordos procurando evaluar como es el proceso enseñanza/aprendizaje en los estudiantes sordos para poder generar programas de apoyo. Los resultados dejan ver que faltan aun aspectos a fortalecer y trabajar como es el hecho que la FCH debe contar con un programa que le permita identificar a los alumnos con NEE, generar apoyos para que el alumno sordo pueda adaptase, compartir su preocupación de rezago e intimidación, proporcionarle la información adecuada sobre el ingreso y acceso a la FCH, así como el trabajo compartido entre los docentes de la facultad y el grupo coordinador encargado de velar por la inclusión. A pesar de que la población docente y administrativa de la facultad tiene una postura de mejorar diferentes aspectos en la atención a la población de estudiantes sordos señantes, su percepción en relación al apoyo que se brindó en ese momento a la estudiante sorda señante para adaptarse en la facultad no fue satisfactoria; de igual manera la mayoría estuvo de acuerdo a que no existe información disponible del trabajo docente en el aula en la facultad para enriquecer con sugerencias a otros docentes, ni se proporcionó información a la estudiante sorda señante para el ingreso y acceso a la facultad, así como no existió en ese momento una figura que representara o tuviera la función de apoyar a la estudiante sorda señante y tampoco existía hasta ese momento un trabajo de grupo coordinado para atender las necesidades de la estudiante. 


\section{Artículos Empíricos}

Tabla 2

Puntajes globales por aspecto evaluado en el personal docente y administrativo

\begin{tabular}{|c|c|c|c|c|}
\hline Aspecto identificados & $\begin{array}{l}\text { Totalmente de } \\
\text { Acuerdo }\end{array}$ & Acuerdo & Desacuerdo & $\begin{array}{c}\text { Totalmente en } \\
\text { Desacuerdo }\end{array}$ \\
\hline 1.-Programa de formación docente & $5 \%$ & $40 \%$ & $30 \%$ & $25 \%$ \\
\hline 2.-Necesario hacer adaptaciones curriculares & $75 \%$ & $15 \%$ & $10 \%$ & $0 \%$ \\
\hline $\begin{array}{l}\text { 3.-La facultad cuenta con un programa de } \\
\text { detección de NEE }\end{array}$ & $0 \%$ & $15 \%$ & $30 \%$ & $55 \%$ \\
\hline $\begin{array}{l}\text { 4.-La facultad es inclusiva y previene la } \\
\text { discriminación }\end{array}$ & $70 \%$ & $30 \%$ & $0 \%$ & $0 \%$ \\
\hline $\begin{array}{l}\text { 5.-Evaluar enseñanza/aprendizaje para dar } \\
\text { soluciones }\end{array}$ & $15 \%$ & $50 \%$ & $10 \%$ & $25 \%$ \\
\hline 6.-Apoyo al alumno sordo para adaptarse & $0 \%$ & $10 \%$ & $25 \%$ & $65 \%$ \\
\hline 7.-Trabajo compartido entre profesores & $0 \%$ & $20 \%$ & $30 \%$ & $50 \%$ \\
\hline 8.-Proporcionar información al alumno sordo & $0 \%$ & $10 \%$ & $45 \%$ & $45 \%$ \\
\hline 9.-Apoyo a los alumnos sordos & $0 \%$ & $5 \%$ & $30 \%$ & $65 \%$ \\
\hline 10.-Trabajo del grupo coordinador & $0 \%$ & $35 \%$ & $50 \%$ & $15 \%$ \\
\hline
\end{tabular}

\section{Discusión}

La inclusión educativa involucra a muchas instancias, por lo que es importante que todas las partes se encuentren sensibilizadas en el trabajo que implica, ya que el propósito final es minimizar o eliminar las barreras que limitan el aprendizaje y promover la participación de todos (Booth \& Ainscow, 2000), esto apoya a lo que reportó la población estudiantil y de docentes y administrativos en las categorías de políticas escolares y cultura escolar inclusiva, donde ya existe en su mayoría conocimiento y sensibilización respecto al tema de inclusión. Aunque ha sido más abordada la inclusión educativa a otros niveles, en México ya existen diferentes instituciones públicas y privadas a nivel superior, lo cual implica y motiva a que en la UAEM se generen nuevas expectativas y propuestas de innovación respecto al tema. Esto apoya lo expuesto tanto por los docentes y administrativos como por los estudiantes oyentes, 
al reportar dentro de la encuesta de que a pesar de que se muestran sensibilizados, se identifica también que aún existen barreras de aprendizaje y la participación en la $\mathrm{FCH}$, faltan cosas por proponer y tomar acciones, como lo referido por algunas investigaciones previas donde se expone que es conveniente superar algunas de las barreras del aprendizaje como los modelos a seguir, la organización de la institución, además de sugerir que es necesaria la investigación en el tema que de un sustento empírico para mejorar el proceso de inclusión, logrando con ello sensibilización de los profesionales educativos y trabajo colaborativo multidisciplinario, donde se mejorarán las estrategias de enseñanza y aprendizaje (Céspedes, 2013; Gutiérrez, 2007; Soto, 2003; Valenzuela, Guillén \& Campa, 2014).

De acuerdo al aspecto de barreras sociales más del 50\% de los encuestados no mostraron la presencia de éstas, por lo que se puede inferir que en la $\mathrm{FCH}$, la inclusión se encuentra en proceso. Tal como lo reporta la población docente y administrativa donde a pesar de que exponen una gran diversidad de necesidades dentro de la facultad, como el que no exista una figura que brinde apoyo al estudiante sordo señante, ni un trabajo coordinado entre personal académico y administrativo para ello, hay disposición y se muestran positivos en que esto puede ser perfectible.

\section{Conclusiones}

La inclusión educativa en México en nivel superior se encuentra en proceso, aún faltan acciones por ejercer; sin embargo, ya se muestran frutos del camino iniciado, lo que promueve a un compromiso en nuestro escenario, en la $\mathrm{FCH}$ y en toda la UAEM, junto con las diferentes instancias a continuar e innovar en esta temática, ya que la inclusión no es solo el proceso de ingreso al nivel superior sino el apoyo en la permanencia de los estudiantes a lo largo de la formación para garantizar su aprendizaje y egreso en tiempo y forma. Esto abre un campo de trabajo en la investigación de las trayectorias escolares de los estudiantes con NEE, así como la implementación de estrategias para favorecerlas. 


\section{Artículos Empíricos}

\section{Referencias}

Andrade, A., Monsalve, T., \& Aristizábal, K. (2015). Alcances del principio de no discriminación de las personas en situación de discapacidad en el ámbito de la educación superior en Colombia. Revista Latinoamericana de Derechos Humanos, 26(1), 99-122.

Booth, T. \& Ainscow, M. (2000). Índice de inclusión. Desarrollando el aprendizaje y la participación en las escuelas. Bristol, UK: Centre for Studies on Inclusive Education (CSIE).

Céspedes, A. (2013). Libro guía para educadores. Camino a la escuela inclusiva. Trastornos del desarrollo desde las neurociencias aplicadas a le educación. Santiago de Chile: Fundación MIRAME.

Cruz, J. (2013). La inclusión de personas sordas en la educación superior. Recuperado de http://noticias.universia.net.mx/ actualidad/noticia/2013/08/05/1040912/ inclusion-personas-sordas-educacionsuperior.html

Delgado, U., Martinez, F., Flores, M \& Lechuga, A. (2016). Inclusión Educativa en América Latina, una mirada más allá de la educación especial. ConCiencia EPG. 1(1), 139-151.
Díaz, Y \& Rodríguez, M. (2009). La enseñanza de la lengua. Educación y Sociedad, 7(2). Recuperado de http://www.culturasorda.org/breve-resena-historica-de-laensenanza-de-la-lengua-a-las-personassordas/\#_ftnref3

Gómez, R. (2014). La inclusión de la persona sorda a la Educación Superior. Revista Iberoamericana de Educación Inclusiva, 8(1), 93-108.

Gutiérrez, M. (2007). Contextos y barreras para la inclusión educativa. Horizonte Pedagógico. 9(1), 47-56.

Jullian, C. (2001). Génesis de la comunidad silente en México. La Escuela Nacional de Sordomudos (1867 a 1886) (Tesis de licenciatura de Historia). Universidad Nacional Autónoma de México.

Lara, D. (2013). Grupos en situación de vulnerabilidad. México: Comisión Nacional de Derechos Humanos.

Lissi, M., Svartholm, K \& González, M. (2012). El enfoque bilingüe en la educación de sordos: sus implicancias para la enseñanza y aprendizaje de la lengua escrita. Estudios Pedagógicos, 38(2), 299-320. 
Ontiveros, I. (2015). Investigación educativa, inclusión de estudiantes sordos a la licenciatura en artes visuales. En V. García., S. Aquino., J. Izquierdo \& P. Santiago (Coords.). Investigación e innovación en inclusión educativa, diagnósticos, modelos y propuestas. (335-360). Durango, México: Red Durango de Investigadores Educativos.

Secretaria de Educación Pública. (2012). Orientaciones para la atención educativa de alumnos sordos que cursan la Educación Básica desde el Modelo Educativo BilingüeBicultural. México: SEP.

Soto, R. (2003). La inclusión educativa: una tarea que le compete a toda una sociedad. Actividades investigativas en educación. 3(1), 1-16.

Universidad Autónoma del Estado de Morelos. (2015). Orientaciones teóricas y metodológicas esenciales para diseñar adecuaciones curriculares en educación superior. Recuperado de http://www.uaem. $\mathrm{mx} /$ sites/default/files/orientacionesteoricas.pdf

Universidad de Guadalajara. (2010). Plan de desarrollo institucional visión 2030.
Recuperado de http://www.udg.mx/ sites/default/files/Universidad\%20de\%20 Guadalajara\%20-\%20Plan\%20de\%20 Desarrollo\%20Institucional\%20-\%20 Visi\%C3\%B3n\%202030-1.pdf

Universidad Tecnológica de Santa Catarina, Nuevo León. (2013). Educación inclusiva. Recuperado de http://www.utsc.edu. $\mathrm{mx} / 02$-edu_incluyente/edu.html

Universidad Tecnológica del Valle del Mezquital del Estado de Hidalgo. (2015). Educación Superior Incluyente. Recuperado de http:// www.utvm.edu.mx/?page_id=1945

Valenzuela, B., Guillén, M. \& Campa, R. (2014). Recursos para la inclusión educativa en el contexto de educación primaria. Infancias Imágenes, 13(2), 64-75.

Victoria, J., Arredondo, J \& Román, J. (2013). Estrategia de inclusión de alumnos sordos en la educación superior, por medio de un diccionario electrónico contable. Presentado en el XVIII Congreso Internacional de Contaduría Administración e Informática. Recuperado de http://congreso.investiga.fca.unam. $\mathrm{mx} /$ docs/xviii/docs/8.10.pdf 


\section{Anexo 1 \\ Cuestionario para estudiantes Inclusión de estudiantes sordos en la UAEM}

\begin{tabular}{|c|c|c|c|c|}
\hline Pregunta & 4 & 3 & 2 & 1 \\
\hline $\begin{array}{l}\text { 1.- ¿La Universidad Autónoma del Estado de Morelos dispone de los medios para } \\
\text { solucionar cualquier tipo de adaptación en el acceso al currículum para los alumnos } \\
\text { Sordos y potenciar sus capacidades? }\end{array}$ & & & & \\
\hline $\begin{array}{l}\text { 2.- ¿La Facultad de Comunicación Humana de la UAEM prevé con pertinencia el } \\
\text { ingreso a personas Sordas a los programas educativos para licenciatura y posgrado? }\end{array}$ & & & & \\
\hline $\begin{array}{l}\text { 3.- ¿La Facultad de Comunicación Humana está preparada para que los alumnos } \\
\text { Sordos puedan desarrollarse en igualdad de condiciones que el resto de los oyentes? }\end{array}$ & & & & \\
\hline 4.- ¿Tener un compañero Sordo se convierte en una preocupación? & & & & \\
\hline $\begin{array}{l}\text { 5.- ¿Los alumnos Sordos requieren ser tomados en cuenta y darles la oportunidad } \\
\text { de alcanzar procesos educativos para mejorar sus condiciones de vida? }\end{array}$ & & & & \\
\hline $\begin{array}{l}\text { 6.- ¿Los docentes de la Facultad de Comunicación Humana apoyan con calidad } \\
\text { cuándo los alumnos Sordos lo requieren? }\end{array}$ & & & & \\
\hline $\begin{array}{l}\text { 7.- ¿El intérprete desempeña el papel de mediador comunicativo entre la comunidad } \\
\text { Sorda y la oyente? }\end{array}$ & & & & \\
\hline $\begin{array}{l}\text { 8.- ¿Se debe tener mayor flexibilidad a la hora de exigir la adquisición de competencias } \\
\text { a los alumnos Sordos para la titulación de la licenciatura? }\end{array}$ & & & & \\
\hline $\begin{array}{l}\text { 9.- ¿Los Sordos tienen carencia de lenguaje, y en consecuencia presentan carencias } \\
\text { cognitivas? }\end{array}$ & & & & \\
\hline $\begin{array}{l}\text { 10.- ¿Para que los alumnos Sordos comprendan y participen de la educación escolar } \\
\text { es imprescindible que los docentes de la Facultad de Comunicación Humana } \\
\text { manejen fluidamente la LSM? }\end{array}$ & & & & \\
\hline $\begin{array}{l}\text { 11.- ¿La presencia del alumno Sordo y de la lengua de señas en la facultad de } \\
\text { Comunicación Humana no amenazan ni al maestro oyente ni a la lengua hablada, } \\
\text { sino que se convierte en la mejor garantía de una eficiente educación? }\end{array}$ & & & & \\
\hline $\begin{array}{l}\text { 12.- ¿Los alumnos Sordos tienen derecho a acceder a la universidad en igualdad de } \\
\text { oportunidades que cualquier otro alumno? }\end{array}$ & & & & \\
\hline 13.- ¿Para mí es importante convivir junto a personas Sordas? & & & & \\
\hline $\begin{array}{l}\text { 14.- - } \text { Es necesario ayudar en exceso a los alumnos Sordos, sin valorar sus } \\
\text { posibilidades y capacidades para facilitar su estancia en la Universidad? }\end{array}$ & & & & \\
\hline $\begin{array}{l}\text { 15.- ¿Es necesario gestionar, para adecuar los programas educativos universitarios } \\
\text { que permitan el desarrollo integral del alumno Sordo? }\end{array}$ & & & & \\
\hline
\end{tabular}




\section{Anexo 2 \\ Cuestionario para docentes y personal administrativo Inclusión de estudiantes sordos en la UAEM}

\begin{tabular}{|c|c|c|c|c|}
\hline Pregunta & 4 & 3 & 2 & 1 \\
\hline $\begin{array}{l}\text { 1.- ¿Considera usted que la Universidad Autónoma del Estado de Morelos da } \\
\text { apertura a programas de formación de docentes especializados en educación } \\
\text { bilingüe para Sordos? }\end{array}$ & & & & \\
\hline $\begin{array}{l}\text { 2.- ¿Considera usted la inclusión de un alumno Sordo a la universidad requiere de } \\
\text { adaptaciones curriculares pertinentes para su evaluación? }\end{array}$ & & & & \\
\hline $\begin{array}{l}\text { 3.- ¿Considera usted que la Facultad de Comunicación Humana cuenta con un } \\
\text { sistema de detección a las necesidades educativas específicas de alumnos con } \\
\text { Sordera? }\end{array}$ & & & & \\
\hline $\begin{array}{l}\text { 4.- ¿Considera usted que la Facultad de Comunicación Humana es inclusiva en su } \\
\text { sistema educativo y previene la discriminación hacia la comunidad Sorda? }\end{array}$ & & & & \\
\hline $\begin{array}{l}\text { 5.- ¿Considera usted que al observar dificultades y limitaciones en el proceso } \\
\text { enseñanza/ aprendizaje se presentan posibilidades de estrategias para solucionarlo? }\end{array}$ & & & & \\
\hline $\begin{array}{l}\text { 6.- ¿Considera usted que existe algún apoyo para el alumnado Sordo que tiene } \\
\text { dificultad para adaptarse, particularmente cuando entra por primera vez en la } \\
\text { FCH? }\end{array}$ & & & & \\
\hline $\begin{array}{l}\text { 7.- ¿Considera usted que existe información disponible del trabajo compartido } \\
\text { entre profesores en el aula así como del sistema educativo general de la FCH, para } \\
\text { enriquecerlo con sugerencias? }\end{array}$ & & & & \\
\hline $\begin{array}{l}\text { 8.- ¿Considera usted que se proporciona al alumno Sordo información básica que } \\
\text { necesita sobre el ingreso y acceso a la Facultad de Comunicación Humana para } \\
\text { que pueda familiarizarse? }\end{array}$ & & & & \\
\hline $\begin{array}{l}\text { 9.- ¿Considera usted que hay personal, con el cual los ylas alumnos Sordos puedan } \\
\text { compartir sus preocupaciones acerca de las situaciones de rezago educativo, } \\
\text { intimidación, etc., de forma que éstos se sientan apoyados? }\end{array}$ & & & & \\
\hline $\begin{array}{l}\text { 10.- ¿Considera usted que ha sido adecuado el trabajo del grupo coordinador en } \\
\text { términos de asignación de tareas, composición del grupo, reparto de trabajo entre } \\
\text { los miembros y la distribución del trabajo a desarrollar por todo el colectivo para } \\
\text { atender al alumno Sordo en la FCH? }\end{array}$ & & & & \\
\hline
\end{tabular}

